\title{
Introduction: Design Innovation Management
}

\author{
Rachel Coopera ${ }^{a}$ Alex Williams ${ }^{b}$, Qian Sun ${ }^{c}$ and Erik Bohemia ${ }^{d}$ \\ aLancaster University \\ ${ }^{b}$ Kingston University \\ ${ }^{b}$ Royal College of Art \\ boughborough University, London \\ DOI: 10.21606/drs.2016.629
}

\section{Introduction}

The aim of this section organised by the Design Innovation Management Special Interest Group with themed track on the Design Policy was to explore Changing Design Policies and Practices.

Design has played a vital role in the development of economies, societies and cultures globally. Governments in nations - such as Korea, Denmark and the UK - have long recognised the contribution design makes towards success and have employed a wide variety of approaches to create environments conducive to design. Different national contexts have called for differing tactics to encourage companies to use design more strategically but have met barriers. Yet research into those policies (defined here as 'political visions into programmes and actions to develop national design resources and encourage their effective use' (Raulik-Murphy, 2014) and their ability to unlock the potential of the design industry to respond to social challenges is both recent and scant.

This section starts by identifying and critically examining national and regional design policies, which guide the interaction of design capacities, seen as a stimulus for economic and social change. Looking to the future, there is significant interest in how design policies may be instrumental in catalysing national responses to global challenges re: poverty, ageing and health; conflict and security; climate change; and in the 'movement of everything' (Cooper, 2015).

The section then moves on to consider how design approaches address this. One of the triggers for, and consequences of, this change is an incorporation of co-design as a process in which designers and users collaborate as 'equals' to develop innovative solutions. The UK Design Council is, for example, advocating the use of co-design methods to support the 
development of practical innovative solutions to social problems such as increased cost of elderly care or tackling child poverty (Design Council, n.d.).

The involvement of users in developing solutions acknowledges that their take up is dependent on the ways users make and negotiate meanings of objects and services (Vossoughi, 2013). Research suggests that a move to incorporate co-design processes will have significant implications on future designers' and researchers' practices (Sanders \& Stappers, 2008). So we proposed to explore the following question: how we design, what we design, and who designs?

This leads to questions as to how we best facilitate, resource, and grow such cooperative practices, in both the cultural and organizational senses. This leads us consider issues of support, co-location and clustering, and ultimately back to consideration of policy, and the extent to which this is/should be top-down (market failure driven) or bottom-up (ecological). Authors contributing to this section consider points such as:

- Emergent trends in design policy

- Understanding how such policies might be embedded within the private, public and service sectors

- The value of design, its dimensions and influences, and how differing design approaches address this

- An exploration of sense-making and meaning within innovation

- Evaluations of participatory methods which facilitate co-design processes

- The challenges for stakeholders within co-design, and the support needs of local communities and start-ups

- The significance of resourcing and clustering, and the implications best practice has on policy formation.

Unpacking this discourse in more detail, the papers are presented under four sub-themes: emergent thinking in design policy; the value of design and how design approaches might address this; the emergence of co-design in addressing social challenges; and the significance of resourcing and clustering.

\section{Emergent Thinking in Design Policy: Wherefore Leadership?}

Sun in her paper titled 'Emerging Trends of Design Policy in the UK' investigates the key organisations involved in developing and delivering policies that impact on design in the UK by reviewing their missions and strategies; from these, she identifies opportunities, challenges and trends. She argues that the proliferation of national design promotion bodies has changed the landscape of design agendas at national, international and organizational levels, particularly where design is considered as an important tool for achieving competitive advantage.

A lack of understanding as to how design adds value at both organisational and national levels has necessitated government interventions informing citizens, companies and public 
organisations about its benefits. However, her paper argues that these interventions should address systemic failures rather than market failures, and calls for a holistic approach to understanding the system of design policy to be adopted. With the shift away from a 'design-centric' approach, there is a need to integrate design into wider innovation systems. Moreover, with substantial growth in interest in design for social and public challenges, there needs to be stronger design leadership in central government. This can be seen in the UK in the increase in design capacity, and commissioning of further, across government through training, aggregation of quality information, and building of capabilities in the design sector itself to respond to such challenges. She cites the emergence of the UK's Policy Lab as marking a fundamental shift from 'policy for design' to 'design of policy', in which design principles and methods are piloted re improving the 'pace, quality and deliverability of policy in the Civil Service'.

Amongst the trends she identifies, three resonate with the discourse presented here - the importance of championing design; the need to integrate design within innovation (albeit not from a design-centric approach); and the growing significance of design for social challenges.

Whicher and Swiatek paper 'Past and Future of Design Policy' develop this theme, identifying that Design is progressively moving up the policy agenda at all levels of governance - local, regional, national and European - with the integration of design into innovation policies and smart specialization strategies, whilst local government are building design capacity by appointing design managers to innovate public services. Terminology has evolved from 'Design Infrastructures' through 'National Design Systems' to 'Design Ecosystems', marking a shift from top-down regulatory environments to ones in which design-driven innovation is instigated from the bottom-up. They propose a Design Innovation Ecosystem construct to examine the interplay between the elements of the system and inform tangible policy action. They perceive design support programmes as becoming more specialised; but signal that design promotion needs to be recognised as a strategic investment, and design adopted as a method for inclusive policy-making. Nonetheless, they recognise that more needs to be done to capture the economic and social value of design, as the associated data is still limited and fragmented.

\section{The Value of Design and How Design Processes Address This}

Bragain the paper titled 'The value of design: an issue of vision, creativity and interpretation' seeks to clarify the value of design, its dimensions and its variables from a fragmented literature, which includes economics, marketing, business, management, value engineering, design domains, social and environmental sustainability. She identifies the difficulty in isolating design from other variables that impact firms' performance, particularly as the measurable results of design only become more evident through time. Assuring the impact for innovative design is an incoherent approach, as is market behaviour forecasting, particularly for disruptive innovations unfamiliar to users. Given that Design Management seeks to identify patterns of 'good' design, past studies have explored organizational culture 
in design-centric firms and the cultural change in perspective as they climb the 'design ladder'. However, it is not clear when and how a non-design-oriented company develops the capacity to absorb design. What are the preconditions/prior knowledge to recognize the value of new information, assimilate it, and apply it? In addressing this, Braga identifies a need to deal with the creative process and tacit knowledge, rather than focusing solely on measurable and visible assets.

Haug's paper 'Coordinating product design with production and consumption processes' partially approaches this by focusing on the importance of coordinating product design with production and marketing processes. To this end, he offers a framework that connects product design to four central processes related to the production and consumption of products and their communication, through a study of 16 empirical examples of commercial failures caused by inadequate alignment of the design function.

In a related approach Kim's paper 'How Companies adopt different Design approaches' explores different types of combined design approach that companies adopt across industrial design and engineering design. He identifies the inherent conflicts between the two groups, due largely to the constraints they impose, and hypothesises the need for a cooperative approach.

In contrast, Chatzakis et al's paper 'A Multilevel Approach to Research 'Obscure' Innovation Processes and Practices' introduce Activity Theory and propose a multilevel framework which aims to reveal obscure practices. Their premise is that organisations struggle to sustain an organic and long-term growth and resilience in increasingly hypercompetitive market conditions because they lack agile practices which better leverage their knowledge. Activity Theory provides a means of analysing social and contextual activities within practice; exploring the roles and activities of members within the NPD process identifies the key influencing factors, and hence where decision-making and value lie.

Thurgood and Lulham in their paper 'Exploring Framing and Meaning Making over the Design Innovation Process' take an alternative, more-semantic approach, focusing on innovation within (or the initiation of new product) meaning and how this results in sustained changes in market behaviour. They articulate a framing model which provides insights into how understanding of problems emerges, meaning changes arise, and what experiences and forms they take. They speculate that the capacity to construct meaning creates new opportunities for a more integrated understanding of the whole innovation process.

Knutz's paper 'Fiction as a Resource in Participatory Design' reverses this association, exploring the relation between participation and fiction. Her study presents three case examples of participatory prototyping that make use of play or games as a means of engaging participants in make-believe to create a design space. Her case studies provide particular insight into areas where such fiction is invaluable: gaining more direct userinvolvement in product development; stimulating critical reflection; and in increasing multistakeholder collaboration. 


\section{The Emergence of Collaboration and Co-Design (in addressing public and social challenges)}

Developing the participatory theme further, Broadley et al's 'From Participation to Collaboration: Reflections on the co-creation of innovative business ideas' suggest that design-led innovation interventions are predicated on establishing complex disciplinary collaborations, and reflect on the effectiveness of different co-design methods to support knowledge exchange, drawing on data collected from sandpit style events. They propose that a more nuanced range of methods, tools and techniques can strengthen multidisciplinary engagement and participation, arguing that such approaches can be enhanced by designers and researchers' shifting focus from co-design methods to supporting collaborative mind-sets in knowledge exchange.

Sune Gudiksen et al's paper 'Bridging Service Design with Integrated Co-design Decisionmaker Interventions' presents a similar treaties, developing the idea of what co-design is capable of. This evolves into something that not only encompasses product and service design, but challenges organisational culture and the mind-set of decision-makers, resulting in more successful embedding of a project within the organisation. Their paper investigates how the development of a new service design project - through integrated co-design interventions - has created a shift in mind-set and viewpoints, enabling design to intervene in and, significantly, gain influence over differing organisational levels.

Chun's paper 'Challenges in Co-designing a Building' also explores the challenges faced in implementing co-design approaches, this time in relation to building design. However, Chun raises concerns as this radically changes how we design, what we design, and who designs. The paper compares participatory approaches to others, and identifies a number of challenges in co-designing a building, including: changes in the role of actors in the design process, and issues around managing conflicts between the interests of different users in a multi-user building project. If co-design approaches are to be successful, she argues that architects need to be able to effectively integrate users' lived experience. Moreover, she questions what kind of skills architects need to successfully co-design a building with users and how that differs to that required in more traditional approaches.

In contrast, Ksenija Kuzmina et al's paper 'An Exploration of Service Design Jam and its ability to foster Social Enterprise' investigate the use of (Service) Design Jams as a means of fostering Social Enterprise - recognised as the building blocks of local economies and communities within the UK. Whilst 'jams' (or co-creation sessions) typically enable individuals to free-think - in the case studied, these are used to identify socially and environmentally focused issues and formulate service solutions - there is a recognition that these solutions require significant nurturing and support. These are crucially reliant on individuals who are already willing to engage in entrepreneurial activity, but few approaches cultivate the willingness and motivation of these individuals to engage. Against this backdrop, the authors propose the combination of collaborative design activity with enterprise workshops as a means of inspiring entrepreneurship. 
This effectively leads our discourse to considerations of resourcing, support infrastructures, and ultimately, clustering.

\section{The Significance of Resourcing and Clustering}

Ylirisku et al's paper titled 'Resourcing in Co-Design' introduce the concept of 'resourcing' to describe the fundamental activity of negotiating the use of what is available for co-design, particularly in unfolding of co-design in complex responsive conversations. The authors focus on how co-designers make use of what is available, and hence, how these are turned into resources. They assert how integral design facilitation is to the unfolding action of codesigning, arguing that the quality of bringing together different stakeholders relies on both the facilitator's and participants' abilities to diversify resourcing - using different kinds of tools for guiding attention, attuning response-sensitivities, structuring presumptions and supporting the generation of ideas. The key issue here is how may facilitators prepare 'greater relevance' for an emerging topic?

Storvang's paper 'Space as Organisational Strategy' looks at this issue from a spatial perspective, identifying a strong link between physical space and its ability to enhance creativity, create change and stimulate interaction. Her investigation explores how space can support organisational strategy, particularly in terms of how space can influence people. The challenge in generating interaction and new relations is an interesting one - with implications for design, design management, management and organisational learning.

The theme concludes with a paper by Tsang et al's paper 'The Making of a Sustainable Cultural and Creative Cluster in Hong Kong', which reviews their experiences in clustering organisations rather than individuals. They consider the setup of creative clusters, against a backdrop in which governments often follow prescriptive models imitating successful practices in other regions, but failing to sustain these clusters. Their paper endeavours to identify the fundamental factors in developing a sustainable cluster in a densely populated city. Rather than considering spatial setting as the most significant factor, they argue that the formation of community and creative happening are integral factors for creative production. They develop a three factor model for the evaluation of sustainable clusters, evaluated through an empirical case study in Hong Kong. They conclude that clusters do not simply refer to the co-location of creative groups or solely describe the geographical location and proximity of similar businesses. Ultimately - in common with Stovang - the generation of synergies (based on cluster, community and creativity) must be the final goal, effectively bringing us back full circle to the consideration of policy, and as Whicher and Swiatek note, a shift the 'Design Ecosystems' in which the conditions for design-driven innovation are instigated from the bottom-up.

\section{References}

Cooper R (2015) Future Cities and Design Imperatives, Keynote Address, International Design Congress, Gwangju, South Korea 
Design Council. (n.d.). The Knee High Design Challenge. Retrieved from http://www.designcouncil.org.uk/what-we-do/knee-high-design-challenge

Raulik-Murphy, G (2014). Design Policy into Practice, WDC Design Policy Conference, Cape Town

Sanders, E. B.-N., \& Stappers, P. J. (2008). Co-creation and the new landscapes of design. CoDesign: International Journal of CoCreation in Design and the Arts, 4(1), 5-18. doi:10.1080/15710880701875068

Vossoughi, S. (2013). A Survival Guide for the Age of Meaning. In R. Martin \& K. Christensen (Eds.), Rotman on Design: The Best on Design Thinking from Rotman Magazine (pp. 55-59). Toronto: University of Toronto Press.

About the Authors:

Rachael Cooper is Distinguished Professor of Design Management and Policy at Lancaster University. She is currently working with The Creative Exchange. She is a member of the FUSE board, a nonexecutive Director of the Future Cities Catapult, and a Lead Expert for the UK Government Foresight programme on the Future of Cities, and is on the Academy of Medical Sciences Working group addressing 'the health of the public 2040'.

Alex Williams is an Associate Dean of Enterprise at Kingston University. He is interested in Design Policy \& its implications in: International Development, New Business Models for the Creative Economy, \& Mechanisms for Knowledge Exchange. He is currently PI on an AHRC-funded mapping of Knowledge Exchange networks.

Qian Sun is a senior tutor in Service Design at the Royal College of Art. Her research interests include Design Management, Design Policy, Design Thinking, and Service Design. She is the Principal Investigator of the 'UK-China Design Policy Network' project funded by the AHRC.

Erik Bohemia is the Programme Director in the Institute for Design Innovation at Loughborough University, London. He is interested in Design as a cultural practice and the material effects of design. He is currently researching the 'construction' of the user and how this guides the design process. 